\title{
Research on Legal Norm Problems of Electronic Evidence Legislation Hierarchy
}

\author{
Xue-Guang WANG \\ Criminal School, East China University of Political Science and Law, shanghai, China \\ wangxueguang@ecupl.edu.cn
}

Keywords: Electronic Evidence, Legislation Hierarchy, Legal Norm

\begin{abstract}
The paper mainly discusses the legal norm problems of Electronic Evidence Legislation hierarchy in China. Based on the analysis and summary of Electronic Evidence Legislation hierarchy, it provides corresponding suggestions and solutions to systematize Chinese electronic evidence legislation on the aspect of perfecting the legislative construction of electronic evidence.
\end{abstract}

\section{Introduction}

The legal system of electronic evidence in China is relatively scattered and confused, both concludes the high effectiveness basic laws such as "electronic signature law", "contract law", "criminal procedural law", "the civil procedure law" and "administrative procedural law" etc., and has local norms of lower rates. There are both the laws reflecting the will of state, and the regulations reflecting the local lawmakers' opinion. Overall, the venation of electronic evidence legislation is similar to the other specialized legislation system, but we can find that high effectiveness of the basic law and administrative rules and regulations in the concrete is relatively lack of measurement criteria of judicial adjudication and the basis of enforcement in the specific judicial practice through the thorough analysist, the lower rates of the administrative rules is constrained by a specific department, the local laws and regulations is confined to a particular region, they have so heavy local characteristics that it is difficult to put into force in the country. So it leads the judicial organ and the inspection organ to fumble ceaselessly in the judicial practice, and it is needed for specific issues to be standard in the form of judicial interpretation, it seems to have formed the circle of stopgaps, let alone to form a basic idea and legal culture for legal problems of electronic evidence. To radically change the situation, we can reconstruct the legislation to regularize the electronic evidence legislation hierarchy.

\section{The Problem of Electronic Evidence Legislation Hierarchy}

The problem of electronic evidence legislation hierarchy mainly embodied on three aspects in China. Firstly, between the different gradations of electronic evidence legislation are lacks of the relevance of close coordination. Secondly, different departments in law enforcement, the rule of law related to electronic evidence used by different departments in law enforcement and administration of justice, such as the standardized process, which is the basis to take the evidence for public security system and accrediting body, exist differences. Thirdly, the standard of the legislation of electronic evidence about forensics tools and techniques is lack of the unity of the law, there are still is fiercely independent.

\section{The Correlation between the Various Levels of the Electronic Evidence Legislation Is Poor}

The related research in the field of electronic evidence in China is still in its infancy, the legislation and practice still have many problems. At present, China has not specialized electronic evidence law, but the revisions of the three major procedural law have established the electronic data as the legal evidence type, so that the real legal conditions is resolved to ensure the electronic data enter into judicial procedure. 
Before revised three major procedural laws, electronic data has been applied to some legislative practice and judicial adjudication, for example, Contract law clearly identified the written form of contract contains the existence form of tangible performance content, such as contract, letters and data message etc., one of them, the data message includes telegram, telex, fax, electronic data interchange and email [1]. The law of electronic signature clearly indicates the data message refers the electronic, optics, magnetism or similar means generated, sent, received, or stored information [2]. The Contract law and the electronic signature law are earlier to rule the electronic data as the evidence form in the level of basic law. In addition, the supreme law, and other joint department also issued corresponding judicial interpretation, rules the specific judicial practice requirements of computer data, for example, article 22 of "the certain provisions of the supreme people's court about civil action evidence" requires to safeguard the primitiveness of computer data. The three departments and two chambers published (the ministry of public security, the ministry of state security, the ministry of justice, the supreme people's court and the supreme people's procuratorate) "some rules of several problems about deal with the examination and judgment evidence in death penalty cases" details the investigatory requirements of electronic evidence.

After revised three main procedural laws, the ministry of public security, the judiciary, (The related departments of judicial authentication) and other relevant departments presented some normative guidance on the investigation practice and judicial application of electronic evidence, for example, article sixty-three of "the procedure stipulation that the public security organ dealed with criminal case", requires the copy of the electronic data shall be attached with a literal interpretation of the production process and the original, original place, and the production process and a literal interpretation of the original, original place, and concerned personnel signature by the producers or holder or unit. The Supreme law has issued the corresponding judicial interpretation on new type of legal evidence of electronic data in the procedural law, for example, "the explanation of some issues of the supreme people's court on implementing the criminal procedure law of the People's Republic of China", requires to audit the authenticity, legitimacy and relevance of electronic data's related evidence, and raises a claim for legislative confirmation of the proof ability and probative force.

Looking at the above related provisions of the three major procedural laws and the revisions on electronic evidence, it is lack of a certain correlation between different levels of law. This correlation means different levels of laws and regulations can form a complete, unified and harmonious legal system aim at some certain legal questions, and all levels of regulations can reflect and perfect each other, and no simple repetitive rules, even contradictory, conflicting rules, they can become a complete legal system. Although the three major procedural laws have identified the electronic data as the legal evidence, they have had no clear explanation for its definition and no the specific applicable regulations of the electronic data. Data message in electronic signature law refers to the information in the electromagnetic light forms. It is too broad that it is fail to reflect digital characteristics of electronic data. In addition, most of the rules of electronic data evidence are formulated by the ministry of public security or by its subordinate institutions, such as "the crime scene investigation of the ministry of public security and the examination rules of electronic evidence" are formulated by public information network security supervision affiliated the ministry of public security. There are serious legal gap between the qualitative of basic law and rules of obtaining evidence, which is not a department regulations, specifically it is lack of electronic evidence laws between the two levels. From the rules established by the ministry of public security, it is too simple to set the rules of evidence on the electronic data, even without distinction, for example, "the program rules for the criminal cases handled by the public security organs" and "the program rules for the administrative case handled by the public security organs", they are completely consistent in the requirement of the electronic data copy, and the formulation of provisions is almost identical. But from the point of requirement proved by a criminal case, it needs to reach the degree to exclude reasonable doubt, so compared with the civil and administrative cases. It should be stricter for the authenticity and legitimacy of evidence. It is the same to the effectiveness of the evidence on the electronic data, but we could not equate the requirement of criminal procedure evidence to the requirement of administrative litigation evidence. 
In terms of provisions related to electronic evidence law, there are very large hierarchy legal vacuum between different levels of the laws and regulations and the same level, it is lack of a relationship in close coordination, at the same time, it doesn't form a unified perfect legal system to rule electronic evidence forensics, and how to affirm the proof ability and the probative force of electronic evidence.

\section{The Relevant Legal Rules of Electronic Evidence Used by Different Departments Are Multilayered}

Although the electronic data has been established as the legal evidence, but the judicial application of electronic evidence in the lawsuit still has many problems. The investigation organ usually conducts some behaviors, such as the search, preservation of evidence, inspection, and appraisal in criminal cases. In civil cases and administrative cases, these behaviors are mostly provided by the parties, if the parties are limited to a certain condition and can't actively seek, they can apply to the court for execution. As a kind of evidence, the electronic data is so. But due to the technology characteristics of electronic data, it is not like traditional evidence which has intuitive feelings on the external performance in many cases. Therefore, it mostly needs a professional analysis by professionals for test analysis of the electronic data. Such as the fixation and the save of electronic data, the implementing subject usually needs to have a digital signature technology, encryption technology, etc., also it requires the implementing subject can skillfully use a lot of technical equipment, in case the primitiveness of data is destroyed, such as read-only lock, copy machine, etc.

The check analysis subject on the electronic data are divided into two kinds, one kind is computer judicial appraiser of judicial authentication institutions, another kind is the technicians or expert witnesses of investigative organization. The rule of law and technology the two kinds of subject adopted in the implementation of a series of operation on electronic data are not completely consistent. Now, the two departments are both trying to set the technical specifications on disposition of electronic data, and these technical specifications are not exactly the same at the name, scope, category, methods and so on. For example, SF/Z JD0400001-2014, the "the general implementation norms of judicial authentication on electronic data " and GB/T 29362-2012 the " search and inspection procedures on data of electronic physical evidence ", the former is to authenticate "electronic data", while the latter is to authenticate " data of electronic physical evidence "; Secondly, there are obvious differences for both of them in the inspection procedure and expression of conclusion. The computer identification of judicial authentication institutions mainly depend on the technology specification of judicial identification, and the investigative organization mainly adopts the technical standards of public security industry.

Whether the analysis and identification of the electronic data done by the investigative organization in the process of investigation, or an authentication of electronic data conducted by appraiser according to relevant rules, they both have some commonness. Firstly, the commonness of the technical attributes on the electronic data: both of them are disposal activities which are based on the characteristics of the electronic data, therefore, there is no essential difference on the implementation of technology; Secondly, the commonness of disposal tools on electronic data: the majority of technical tools used by the appraiser and technicist should be the same or similar, and the analysis and results based on the same or similar actual forensics and critical tools, have a certain degree of similarity. Therefore, in order to the unity of legal rules on electronic evidence forensics, it is necessary for relevant departments to work together, design and programme unified rules of evidence law, and apply to the investigative activities and appraisal activities of evidence, so that the effectiveness and accuracy of different departments in evidence collection, inspection, analysis of electronic evidence can check each other.

\section{Legislation of Electronic Evidence on Forensic Tools and Technical Standards Are Lack of an Unified Legal Provisions}

In addition to clarify the legal status of legislation on electronic evidence, it is more important for the forensics, verification and certification of electronic evidence. It is the problem at home and 
abroad about how to effectively apply electronic evidence to judicial practice. Electronic data forensics is the key step to apply electronic data evidence to suit, but the electronic evidence forensics has not formed unitive systemic specification on tools and techniques [3-5].

Electronic data forensics is a very rigorous course, any fault operation could lead to the loss of evidence. Therefore, any step in the process of forensics must accord with the requirement of legal proceedings, we shall strictly regulate the tools and techniques involved forensics. As the reason as the process of standardization of electronic evidence legislation in China starts late, many laws and regulations is not very complete, most of them is principle requirements, and lack of maneuverability, such as "the program rules for the criminal cases handled by the public security organ", it puts forward the request for copies of electronic evidence, but there is no specific treatment process. At the point of evidence specification, the specific using tools, techniques and detailed procedure of evidence-obtaining rely mainly on industrial standards and technical specifications. Such as GB/T29362-2012 "the inspection procedures on the search of electronic physical evidence data" established by the national standardization management committee, GB/T29360-2012 "the inspection procedures on the recovery of electronic physical evidence data"; SF/Z JD0400001-2014 "General implementation specification on judicial authentication of electronic data" established by the ministry of justice; GA/T 976-2012 "common methods on forensic science appraisal of electronic data" set by the ministry of public security, etc. However, after carefully analyzing the industrial standard or technical specification, we can find they are lack of integrality in the system, most of them are scattered, individual, and lack of practical operability. In addition, in the process of forensic analysis and identification, there are no detailed instructions on what tools and which kind of technic can be chosed. This can lead to some cases which don't conform to the requirements of forensics in the judicial practice, for example, some people use pirated software and old version of the forensic tools to obtain evidence and so forth.

\section{Suggestions on Systematizing Electronic Evidence Legislation Hierarchy}

For systematizing electronic evidence legislation hierarchy in China, we can rule and adjust the system structure of legislation on electronic evidence wholly and uniformly by perfecting the structure. Combining with the station of relevant legislation on electronic evidence in China, we can perfect the construction of electronic evidence legislation as follows.

\section{To Perfect the Legislative Model of Electronic Evidence}

Legislation of electronic evidence is a comprehensive and long-term work, the hope that is to solve all the problems by drafting a law is not realistic. This requires us to carry out the research on the programme of electronic evidence legislation, deep research the requirements of electronic evidence legislation, reasonably allocate the legislative resources, and formulate a long-term plan to establish a comprehensive, coordinated and orderly system of laws and regulations on electronic evidence. We should start from constructing rules of evidence, and gradually consolidating foundation, we can draw lessons from the experience of legislation of electronic evidence in USA: firstly, we need to come on unitive evidence rules, which play a guiding role, aim at the overall evidence type, and then to gradually make a more comprehensive unified rules of electronic evidence, at the same time, put forward a more comprehensive and professional law on electronic evidence. For example, the law of electronic evidence forensics mainly refers to "the Federal Rules of Evidence" in USA. And "the Federal Rules of Evidence" doesn't issue different evidence rules with the other types of evidence. Both the electronic evidence and other evidence are to accept the test of the same forensics and the rules of identification in judicial proof. But electronic evidence is after all different from the traditional type of evidence. Thus, "the Unified Evidence Rule in 1999 in USA"," the law of the uniform computer information in USA", and "American uniform electronic transaction" formulated more appropriate rules on the electronic evidence forensics according to the characteristics of information technology on electronic evidence. Secondly, it is necessary to strengthen the evaluation on the implementation of laws and regulations about electronic evidence. In China, we should firstly 
evaluate the station of implementation and the effect of implementation about electronic evidence relevant laws and regulations, then timely modify or amend the relevant legislation of electronic evidence [6-8].

\section{Refining All Aspects of Provisions Relating to Electronic Evidence}

Legislation of electronic evidence needs detailed stipulation at its definition, characteristics, types, the principle of forensics, the forensics model, forensics technology, forensics tools, the forensics subject, the forensics process and so on, to refine the requirements involving electronic evidence. We can take example by from the process of general evidence conversion. In the process of case investigation and judicature, the first thing is to obtain evidence. The electronic data is also one of the evidence materials presented by objective carrier. The second thing is to rule out illegal type of evidence depending on the carrier form, electronic data is the legal evidence type specified by the Procedural Law, it conforms to the requirements of the substantive legitimacy; Finally, it need to analyze the connection degree of core evidence and the case facts, according to the rules of certification of electronic evidence, if it is verified, the connection degree can be used as verdict proof. In a word, we should refine the forensics rules, verification rules and certification rules of the electronic evidence.

\section{To Further Perfect the Matched Law about Electronic Evidence}

Based on the theory of legal system design, the legal system and the main module of law are composed of the following submodular, which can connect with each other and exist independently. Therefore, on the basis of perfecting the basic law of electronic evidence, we should clear the specific rules of electronic evidence. In China the Constitution is the fundamental law of the state, the basic law is followed to replenish, improve, concrete implement the requirements ruled by constitution. The State Council set up a system of administrative rules and regulations to lead administrative work, each department according to their own responsibility to make a more detailed rules and regulations. So, we need perfect the legal system of electronic evidence in the legislation of electronic evidence system, briefly, on the basis of building the basic regulations or provisions on electronic evidence we should uniformly design the general principles of electronic evidence, and then get a handle on the rules and other important aspects involving electronic evidence. Since then, the various departments, according to their respective duties, in accordance with the rules and regulations of basic law and administrative, and the difference in the degree of electronic evidence regulations, formulate appropriate detailed rules. Finally, the Supreme People's Court and the Supreme People's Procuratorate shall come on a judicial interpretation on the applicable related issues of electronic evidence to improve the judicial and law enforcement in terms of judicature.

\section{Strengthen the Construction of the Technical Standards on Electronic Evidence Forensics, In Order to Match the Specific Specification of All Departments}

The technical standard of related rules on electronic evidence forensics is the combination foundation of technical and legal. To endow national will to technical standards, and make sure it's compulsory legal effect is concerted action from country to country. Investigation rules of electronic evidence involves the inquisition of electronic data environment, the fixation of equipment, the search and seizure, the retrieval and analysis of information system, and so on a series of technical standards. After perfected the relevant technical standard, related departments should match the other departments with specific duties, such as the ministry of public security, judiciary, to close unite the technical standard and specification of specific departments together, and then make sure the disposal of electronic evidence implemented by each department is more operable.

\section{Conclusions}

The paper mainly studies the legal norm problems of Electronic Evidence Legislation hierarchy in China. Based on the analysis and summary on Electronic Evidence Legislation hierarchy, such as the 
correlation between different levels of the legislation of electronic evidence, the hierarchy of legal rules on electronic evidence used by different departments, whether the legal rules on forensic tools and technical standards of legislation of electronic evidence is unitive, the paper provides corresponding suggestions and solutions to guarantee the legislation of Chinese electronic evidence systematic on the aspect of perfecting the legislative construction of electronic evidence.

\section{Acknowledgments}

This work is supported by National Social Science Foundation of China (No. 11BFX125), PuJiang Talent Project, Peak of law subject construction and Public Security Discipline Construction Foundation.

\section{References}

[1] The article 11 of "Contract Law of People's Republic of China".

[2] The article 2 of "Electronic Signatures Law of People's Republic of China".

[3] P.X. Liu, Study on Creating Chinese Electronic Evidence Rules, China Renmin University Press, 2005, pp.89-93.

[4] Z.W. Nie, Ton the Regulation of the Rules of the Force and the Qualification of Evidence, Journal of SWUPI(Southwest University of Political Science and Law), 2007(2).

[5] F.K. Dai, The Question of The legality toward The Criminal litigation Evidence Attribute, Law Science, 1987(5), pp.22-23.

[6] Michael J. L. Turner, ed. Computer Evidence. Accessed by May 23, 2016. http:// www.computerevidence.co.uk/.

[7] E.Q. Li, Study On Subsidiary Information of Network Evidence, CHINESE CRIMINAL SCIENCE, 2009(4), pp.73-77.

[8] Y.H. Mai, Electronic Data Judicial Forensics Practice, Law Press, 2011, p.33. 